\title{
A WBAN for Human Movement Kinematics and ECG Measurements
}

\author{
Ahmed Baraka, Ahmed Shokry, Ihab Omar, Saged Kamel, Tarek Fouad, \\ Mohamad Abou El-Nasr, Heba Shaban \\ Arab Academy for Science, Technology \& Maritime Transport (AASTMT), Alexandria, Egypt \\ Email: hshaban@vt.edu
}

Received April 1, 2012; revised May 10, 2012; accepted May 26, 2012

\begin{abstract}
Biomedical applications of body area networks (BANs) are evolving, where taking periodic medical readings of patients via means wireless technologies at home or in the office will aid physicians to periodically supervise the patient's medical status without having to see the patient. Thus, one important objective of BANs is to provide the doctor with the medical readings that can be collected electronically without being in close proximity to the patient. This is done through the measurement of the patient's physiological signals via means of wearable sensors. This paper investigates wireless BAN cooperation via actual measurements of human movement kinematics and electrocardiogram (ECG), which are believed to provide patients with easy healthcare for continuous health-monitoring. The collected information will be processed using specially designed software, which in turn will enable the patient to send a full medical chart to the physician's electronic device. In this way, physicians will have the ability to monitor their patients more efficiently.
\end{abstract}

Keywords: Body Area Networks (BANs); Electrocardiogram (ECG); Human Gait; and Movement Kinematics

\section{Introduction}

Body area networks (BANs) are the systems of sensors/ devices that cooperate in close proximity to a person's body to provide a benefit to the user. There are multiple applications of BANs including medical and non-medical applications. Recently, wireless technology has invaded the medical area of BANs with a wide range of capabilities. These applications typically use biomedical sensors to monitor the physiological signals of patients, such as electrocardiogram (ECG), blood oxygen level, blood pressures, blood glucose, body weight, heart rate, oxygen saturation, etc [1-6].

Wireless technology enables clinicians to monitor their patients' remotely and give them timely health information and support. Especially, in emergency situations, real-time health parameter is crucial. According to the American Heart Association, treatment of a patient experiencing ventricular fibrillation within the first $12 \mathrm{mi}-$ nutes of cardiac arrest brings a survival rate of $48 \%$ $75 \%$. On the other hand, long-term health-monitoring requires intensive and repetitive assessment that could last for months or even years to regain the lost functions, such as in the case of rehabilitation. Thus, one of the main challenges in such a case is being able to monitor patients for long-times in domestic environments. BANs provide a promising solution for such situations, however currently, BAN technology is emerging, and there are a lot of problems to address. One of the key challenges associated with BANs is the integration and coordination of multiple sensors with different applications [1-6].

This paper's aim is to investigate wireless BAN cooperation for human movement tracking and ECG measurements, which are believed to provide patients with easy healthcare for continuous health-monitoring. In addition, taking periodic medical readings at home or in the office will aid physicians to periodically supervise the patient's medical status without having to see the patient via means wireless technologies. The collected measurement data will be processed using specially designed software, which will help sending a full medical record of the patient to an electronic device in the acquisition of the physician using wireless technology. Figure 1 shows a schematics diagram of the implemented WBAN. We consider a WBAN that uses wireless wearable sensors for gait kinematics and ECG measurements. The proposed WBAN is assumed to use commercially available noninvasive wireless sensors, as will be shown in detail in later sections.

This paper is organized as follows. Section 2 explains gait analysis, and gives a brief overview of its types and measurement parameters. Then, Section 3 provides a short overview of ECG. Section 4 describes the actual measurements. Future work is provided in Section 5, and 


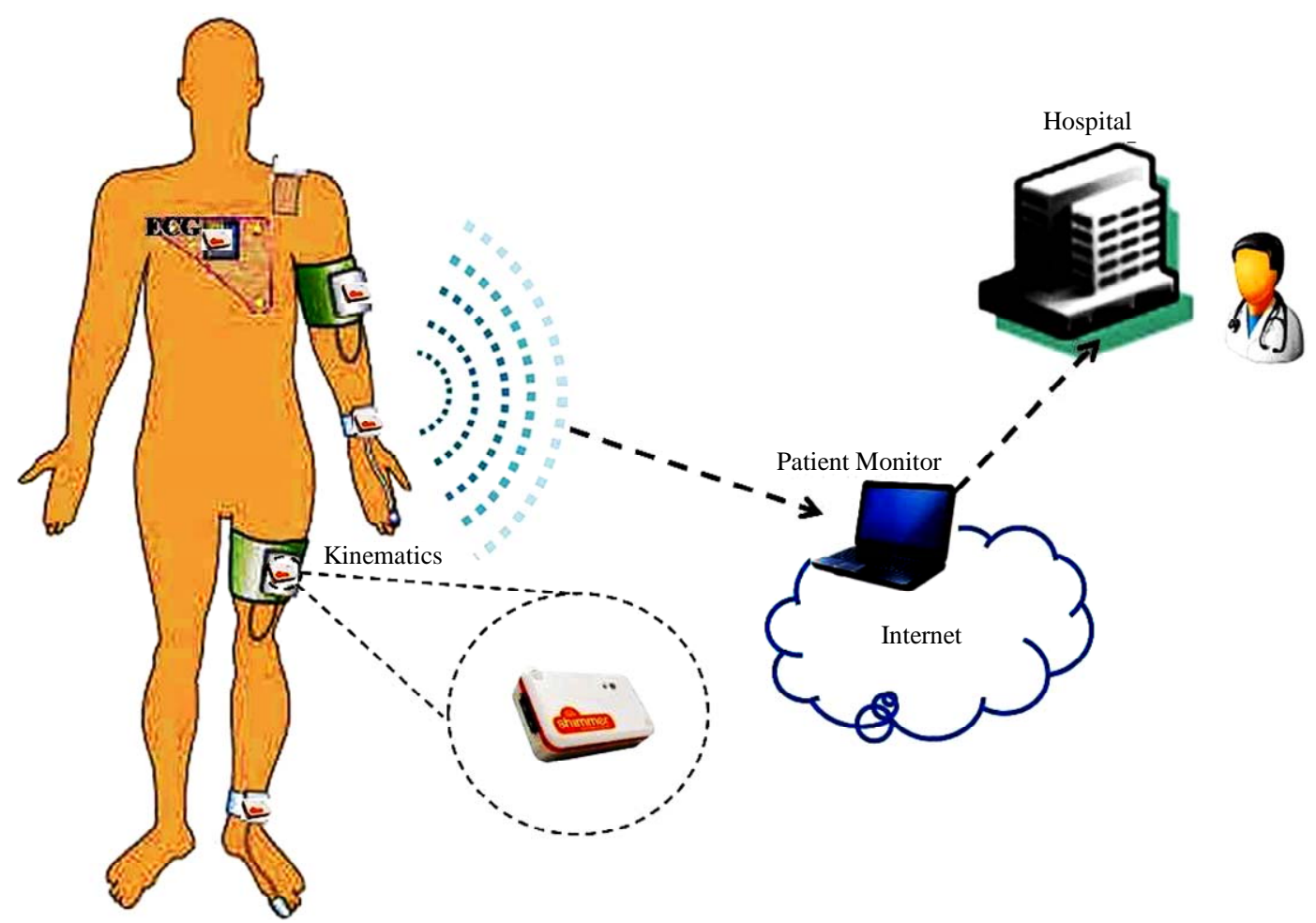

Figure 1. Schematic diagram of the implemented WBAN.

conclusions are given in Section 6.

\section{Gait Analysis}

Gait analysis is the systematic study of human motion. Gait analysis is divided into observational and quantitative gait analyses. Clinical gait analysis is to assist physicians in treating walking disorders.

Clinical gait analysis, also referred to as quantitative gait analysis, is the measurement of gait characteristics, where abnormalities in gait are identified, and the causes are postulated, such as pain, injuries, etc. Accordingly, treatments are proposed. Recently, gait analysis has also received increased attention and been widely used in sports [1-6].

\subsection{Observational Gait Analysis}

Observational gait analysis is primary clinical tool used by clinicians for observing walking patterns, and abnormalities in gait. It is sometimes preferable to physicians than quantitative gait analysis, but it can be very unreliable due to the lack of measurement instruments [1-3].

\subsection{Quantitative Gait Analysis}

Quantitative gait analysis is generally considered to be the best way used to measure walking performance, where accurate measurement devices are included, and gait parameters are calculated. The procedure can be very simple, and is limited to measuring step length with a ruler, or determining speed with a stopwatch. It can also be a very sophisticated process that includes full-body motion capture (MoCap) with very accurate instrumentation, and well-equipped laboratories with experienced personnel. Regardless of the complexity of the method used, the collected measurement data is used to assess the quality of gait, and characterize the locomotion [2-6].

Quantitative gait analysis used to fully describe a person's gait generally includes the measurement of a set of parameters namely, temporal-spatial parameters, kinematics and kinetics. Temporal-spatial parameters generally include the measurement of the parameters related to walking distance, speed, etc. Whereas, kinematics is concerned with measuring the parameters related to the geometry of motion, such as joint angles. Finally, kinetics measures motion parameters that include forces, such as joint moments [2-9].

\section{Electrocardiogram (ECG)}

Electrocardiogram (ECG) is defined as the test that records the electrical activity of heart using electrodes attached to the skin, and recorded by an external device. The typical purposes of using an ECG device are as follows. First, check the heart's electrical activity. Second, determine the cause of unexplained chest pain, which could be caused by a heart attack. Third, find the cause of symptoms of heart disease. Fourth, check if the walls of 
the heart chambers are too thick. Fifth, check the effectiveness of certain medicines. Finally, check the efficiency of mechanical devices that are implanted in the heart, such as pacemakers are working to control a normal heart beat [10].

\section{Actual Measurements}

This section describes the actual measurements of the implemented WBAN for medical applications. The implemented WBAN includes body kinematic and ECG measurements via wireless sensors. It is believed that the measurement of body kinematics and ECG will help to better understand movement artifacts. Moreover, using wireless sensors connected via means of WBANs will help monitoring patients for long periods, which are essential in the cases that require long-term monitoring, such as rehabilitation.

The used sensors are manufactured by SHIMMER, which are low-power wireless sensors specially designed for noninvasive biomedical research. In our motion capture measurement, we use SHIMMER's wireless ninedegrees-of-freedom (9DoF) sensors, shown in Figure 2(a), and for ECG measurements, we use SHIMMER's wireless ECG sensor, shown in Figure 2(b). The measurement setup and captured data will be explained in detail in the following subsections.

\subsection{Gait Measurements}

In the implemented WBAN, gait measurements are taken via SHIMMER's 9DoF kinematic sensor [11], which is equipped with an accelerometer, magnetometer, and gy-

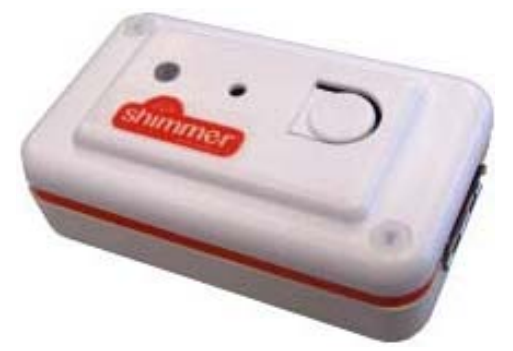

(a)

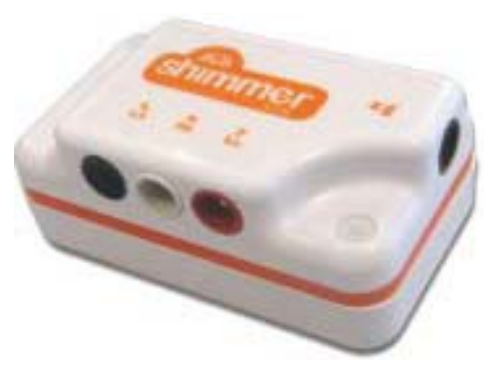

(b)

Figure 2. (a) Wireless 9 DoF sensor by SHIMMER; (b) Wireless wearable ECG sensor by SHIMMER. roscope. In our measurement setup, we consider different measurements for the arms and legs. Figures 3(a) and (b) show the measurement setup for the wrist and leg-shank, respectively. Figures 4(a) and (b) display the captured signals from the arm and leg, respectively. The second measurement scenario included a measurement set of the left and right upper-arm segments, and the measured signals have also been recorded. The measurement setup for the left and right upper-arms are shown in Figures 5(a) and (b), respectively. The corresponding captured signals are depicted in Figure 6.

\subsection{SHIMMER Connect and MATLAB Software}

We use two software packages for captured signal display and processing. For real-time display and data saving purposes, we use SHIMMER connect software package developed by SHIMMER [12]. A snapshot of the program is depicted in Figure 7. Also, we use MATLAB software [13] for real-time data display, and post-processing simulations.

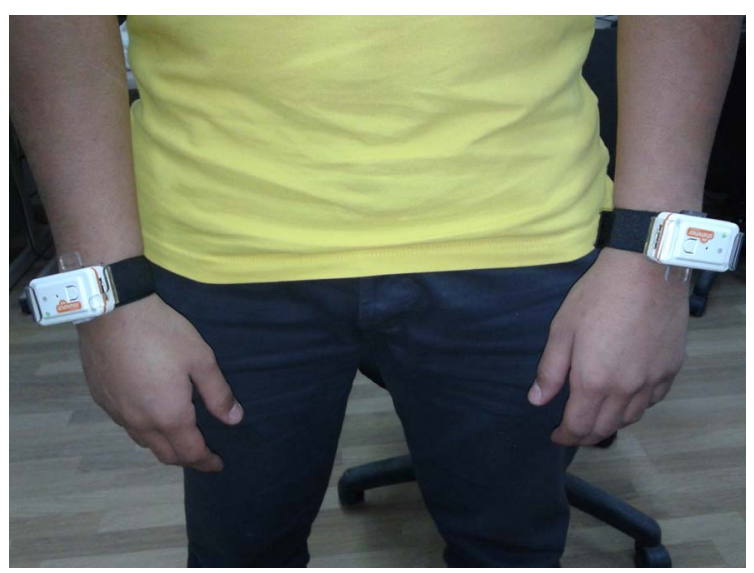

(a)

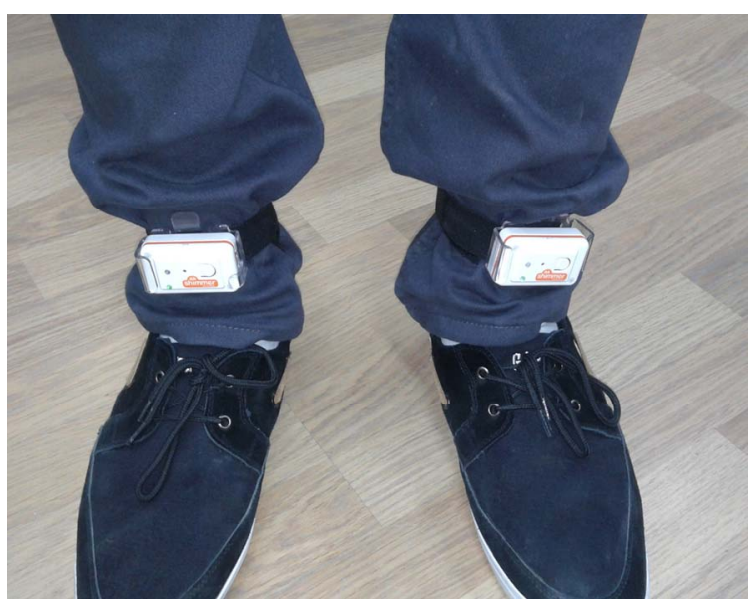

(b)

Figure 3. (a) Wrist kinematic measurement setup; (b) Legshank kinematic measurement setup. 


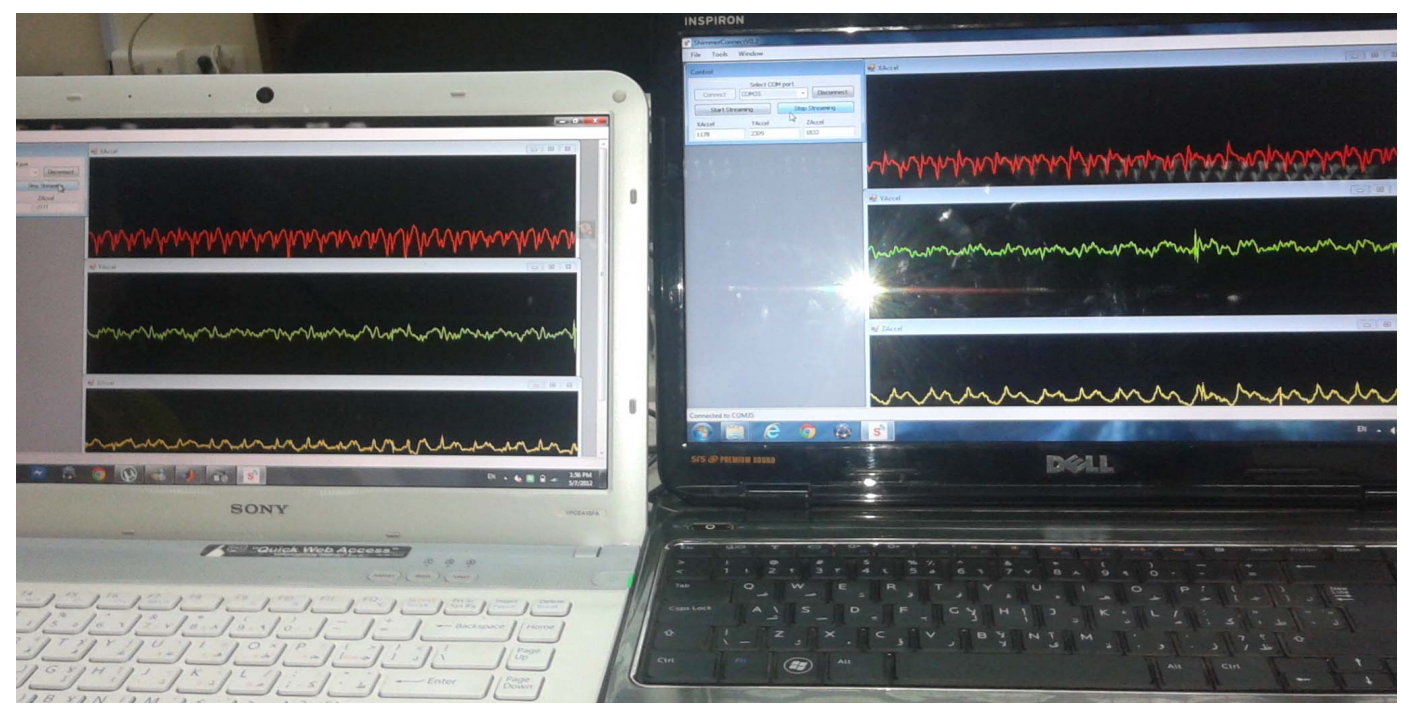

(a)
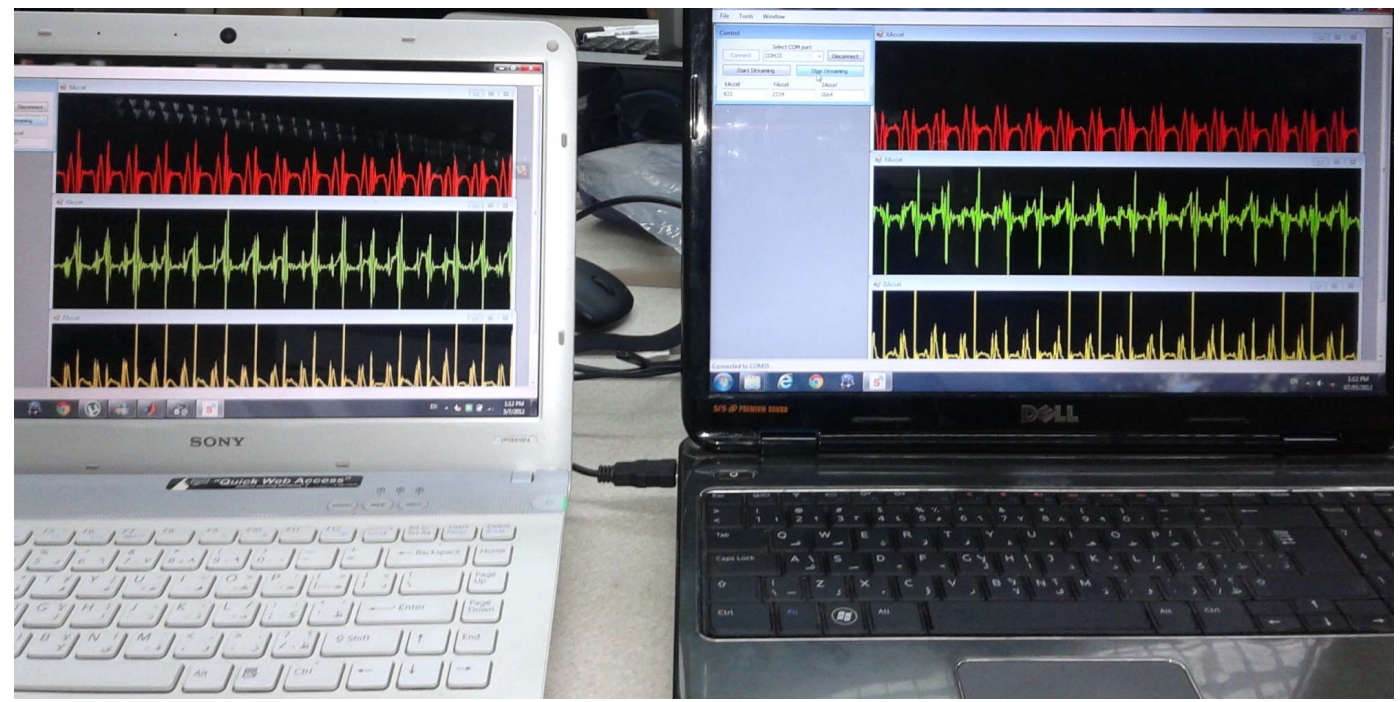

(b)

Figure 4. (a) Display of real-time captured data from the wrist measurements via SHIMMER connect; (b) Display of realtime captured data from the leg-shank measurements via SHIMMER connect.

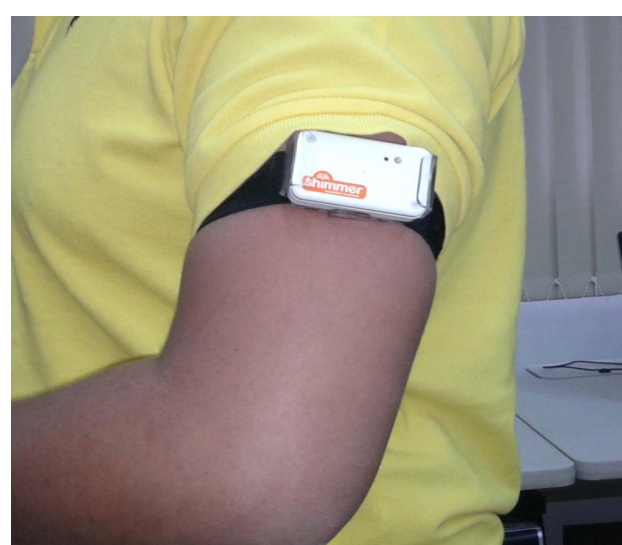

(a)

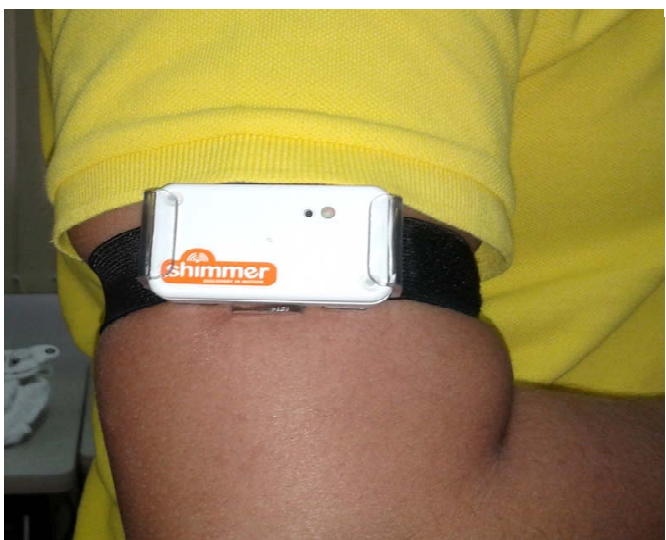

(b)

Figure 5. (a) Left upper arm kinematic measurement setup; (b) Right upper arm kinematic measurement setup. 


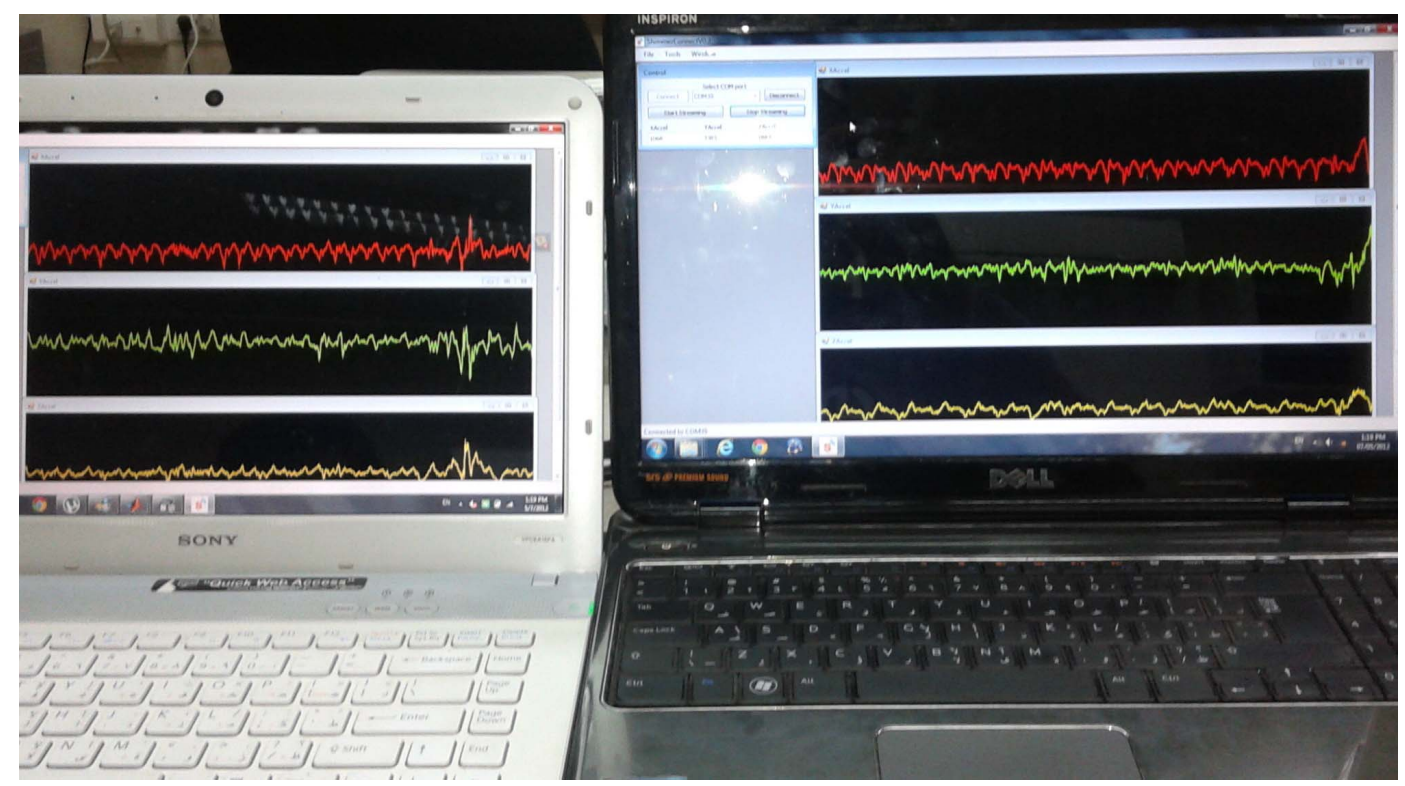

Figure 6. Display of real-time captured data from the left and right upper arm measurements using SHIMMER connect.

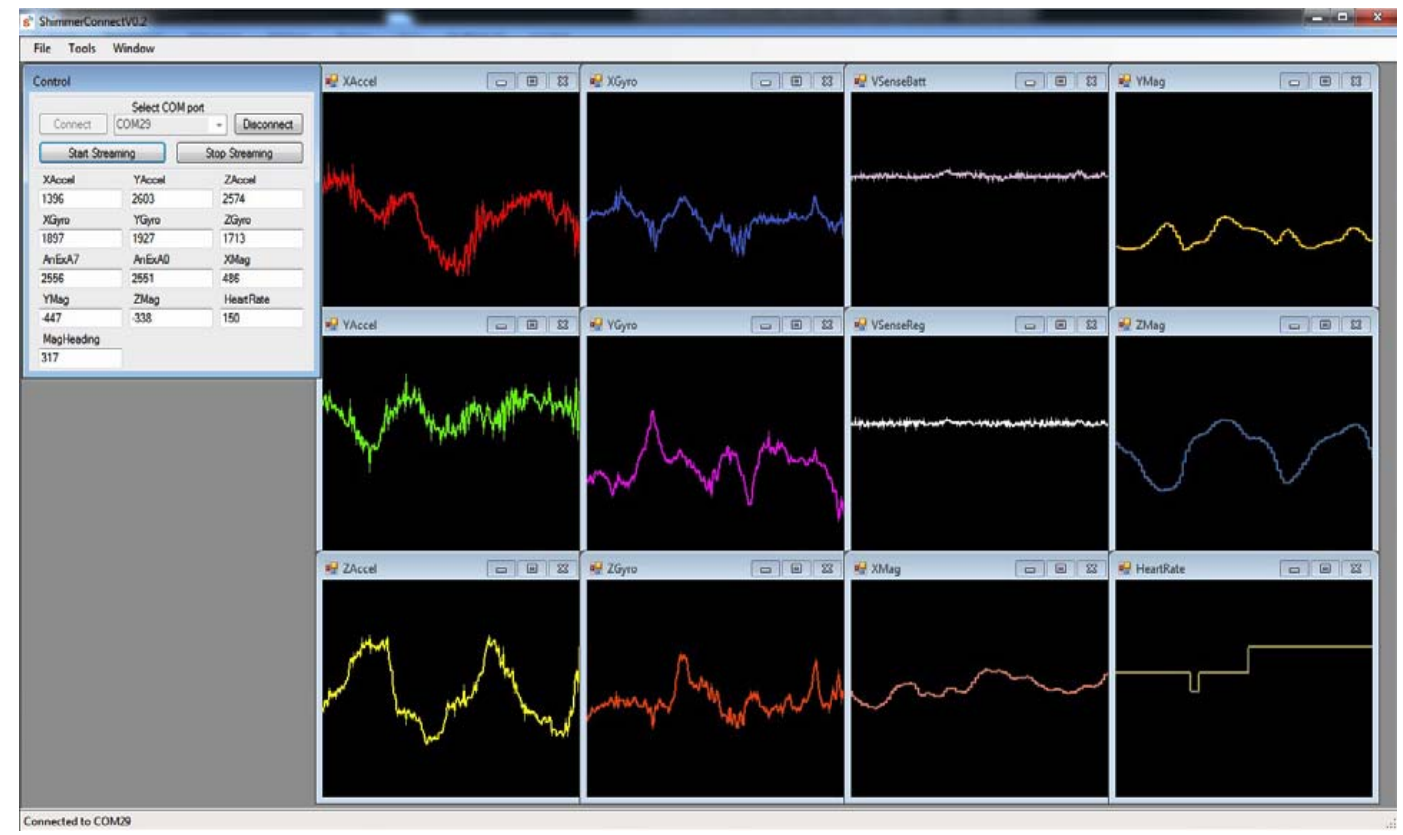

Figure 7. SHIMMER connect software.

\subsection{ECG Measurements}

For physiological signal measurement, particularly for ECG measurement, we use SHIMMER wireless ECG sensor. We further display the measured data using MATLAB for future post-processing simulations. Figure 8 shows a sample captured ECG signal. The captured signal is associated with normal walking speed.

\section{Future Work}

The future work of this project will include the assess- ment of measured data via the implemented WBAN, and integrating the measured gait kinematic and ECG signal into finding more accurate results related to both gait analysis and ECG measurements.

\section{Conclusions}

A WBAN network that includes the measurement of human motion kinematics and ECG signals was implemented via means of wireless noninvasive sensors. The WBAN is suitable for real-time data capturing and processing. The implemented WBAN is ultimately suitable 


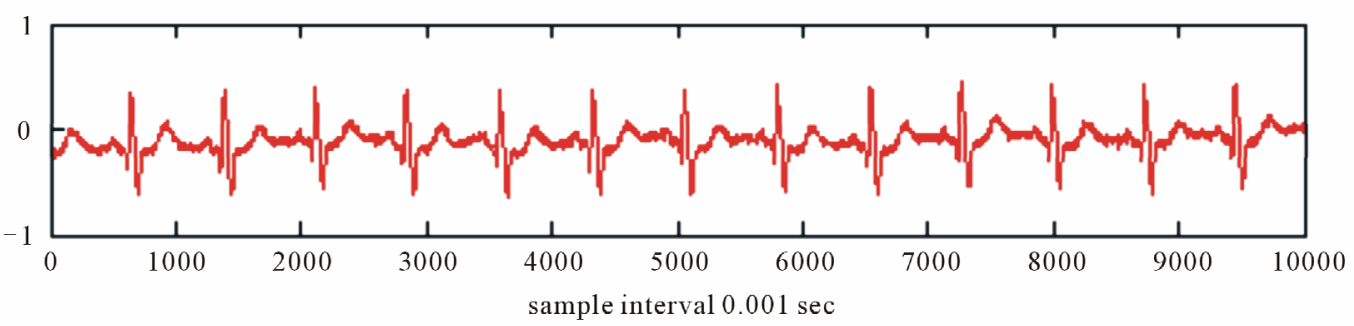

(a)

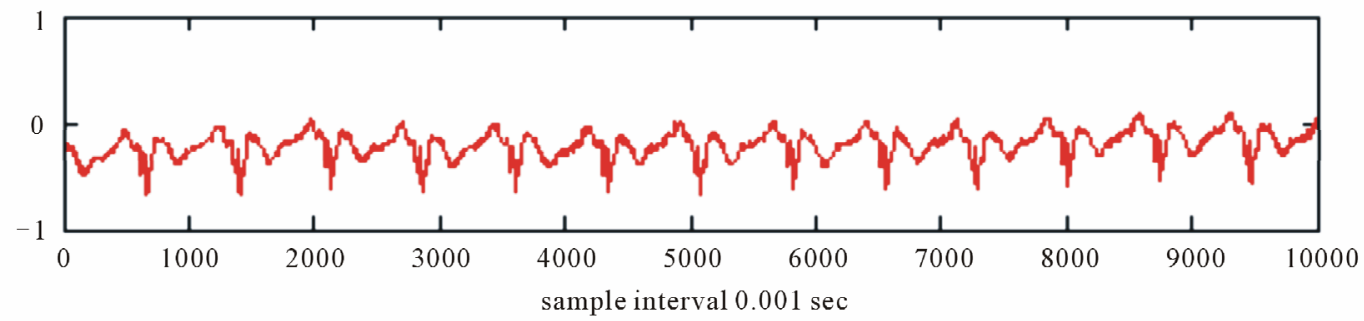

(b)

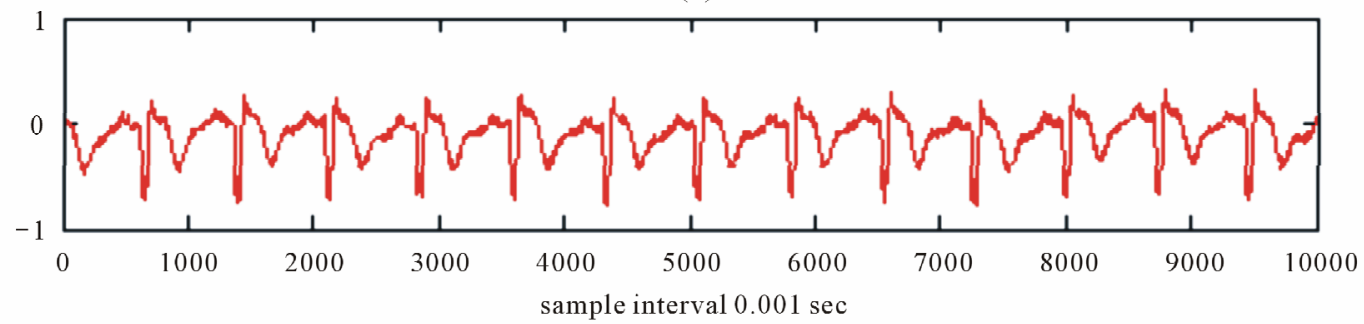

(c)

Figure 8. Captured ECG signal using MATLAB.

for long-time health monitoring and for taking daily measurements at home or in the office.

\section{REFERENCES}

[1] L. D’Astous and B. MacWilliams, "Current Challenges in Clinical Gait Analysis,” ASB 29th Annual Meeting, Cleveland, 31 July-5 August 2002, p. 946.

[2] A. Leardini, L. Chiari, A. Cappozzo and U. Della Croce, "Human Movement Analysis Using Stereophotogrammetryc Part 1: Theoretical Background," Gait and Posture, Vol. 21, No. 2, 2005, pp. 186-196. doi:10.1016/S0966-6362(04)00025-6

[3] H. Shaban, “A Novel Highly Accurate Wireless Wearable Human Locomotion Tracking and Gait Analysis System via UWB Radios,” Ph.D. Thesis, Virginia Tech, Blacksburg, 2010.

[4] H. Shaban, M. Abou El-Nasr and R. M. Buehrer, "Performance of Ultralow-Power IR-UWB Correlator Receivers for Highly Accurate Wearable Human Locomotion Tracking and Gait Analysis Systems,” IEEE Global Telecommunications Conference, Honolulu, 30 November-4 December 2009, pp. 1-6.

[5] H. Shaban, M. Abou El-Nasr and R. Buehrer, "Toward a Highly Accurate Ambulatory System for Clinical Gait Analysis via UWB Radios,” IEEE Transactions on Information Technology in Biomedicine, Vol. 14, No. 2, 2010, pp. 284-291. doi:10.1109/TITB.2009.2037619
[6] H. A. Shaban, M. Abou El-Nasr and R. M. Buehrer, "Reference Range Correlation (RRCR) Ranging and Performance Bounds for on-Body UWB-Based Body Sensor Networks," Progress in Electromagnetics Research B, Vol. 35, 2011, pp. 69-85. doi:10.2528/PIERB11082212

[7] M. Abou El-Nasr, H. A. Shaban and R. M. Buehrer, "Key Design Parameters and Sensor-Fusion for Low-Power Wearable UWB-Based Motion Tracking and Gait Analysis Systems," Progress in Electromagnetics Research Letters, Vol. 29, 2012, pp. 115-126. doi:10.2528/PIERL11120303

[8] M. Abou El-Nasr, H. Shaban and R. Buehrer, "LowPower IR-UWB Coherent TOA Estimators with Suboptimal Sinusoidal Templates for UWB-Based Body Area Networks," Wireless Networks, Vol. 17, No. 7, 2011, pp. 1641-1648. doi:10.1007/s11276-011-0369-0

[9] H. Shaban, M. Abou El-Nasr and R. Buehrer, "Toward a Highly Accurate Ambulatory System for Clinical Gait Analysis via UWB Radios," IEEE Transactions on Information Technology in Biomedicine, Vol. 14, No. 2, 2010, pp. 284-291. doi:10.1109/TITB.2009.2037619

[10] T. Pawar, N. S. Anantakrishnan, S. Chaudhuri and S. P. Duttagupta, "Impact Analysis of Body Movement in Ambulatory ECG,” 29th IEEE EMBS Annual International Conference, 23-26 August 2007, PP. 5453-5456.

[11] A. Burns, B. R. Greene, M. J. McGrath, T. J. O’Shea, B. Kuris, S. M. Ayer, F. Stroiescu and V. Cionca, "SHIM$\mathrm{MER}^{\mathrm{TM}}$ —A Wireless Sensor Platform for Noninvasive Biomedical Research,” IEEE Sensors Journal, Vol. 10, 
No. 9, 2010, pp. 1527-1534.

doi:10.1109/JSEN.2010.2045498

[12] Shimmer Discovery in Motion Software, 2012. http://www.shimmerresearch.com/download/softwares

[13] Mathworks MATLAB and SIMULINK for Technical Computing, 2012. http://www.mathworks.com/ 